\title{
Effect of humidity on optical fiber distributed sensor based on Brillouin scattering
}

\author{
Carlos A. Galindez ${ }^{*}$, Francisco J. Madruga, M. Lomer, A. Cobo and Jose M. Lopez-Higuera \\ Photonics Engineering Group, ETSII y de Teleco, University of Cantabria, \\ Av Castros s/n, 39005-Santander, Spain
}

\begin{abstract}
In real sensors, the crosstalk or undesirable crossed sensitivities must be minimized. Distributed Brillouin sensing is a very useful technique to measure fluctuations of temperature along an optical fiber. However, the later measurement can be influenced by the humidity on the fiber; therefore its effect must be minimized. Because the aforementioned, the Brillouin frequency changes with the humidity. Thus, for a given temperature on a distributed fiber sensor such variations have been investigated. The experimental results obtained using three different types of single mode fibers with $1000 \mathrm{~m}$ length, at $25^{\circ} \mathrm{C}$ are reported in this paper.
\end{abstract}

Keywords: Brillouin shift, relative humidity, distributed sensor, temperature sensor.

\section{INTRODUCTION}

In the field of optical fiber sensors, those distributed based on Brillouin backscattering are useful to control and measure the temperature or the strain applied along an optical fiber ${ }^{1}$. This technique uses the natural Brillouin frequency of any material that is linearly dependent on temperature and strain of the material ${ }^{2}$. Interaction between the scattering and light fields can be described by steady state coupled-intensity equations for a slowly varying amplitude approximation. The Brillouin coefficient is defined for these coupled equations and depends on physical parameters of material such as, density, refractive index, longitudinal elasto-optic coefficient among others ${ }^{3,4}$. Hence, it is not difficult to think that the physical phenomenon of interaction between the light fields into the fiber can be modified by the humidity.

The effect of the humidity on the Brillouin scattering phenomenon is usually not considered; but in this paper, it is reported the results obtained from a systematic experimental study in the laboratory. Along the next paragraphs, the used setup is explained; obtained results are presented and discussed. Finally, the main conclusions are summarized.

\section{EXPERIMENTAL SETUP}

The setup is depicted in Fig. 1. It is composed by a diode laser source, a LiNbO3 electro-optic modulator, an Erbium Doped Fiber Amplifier (EDFA), a fiber WDM device, the fibers under test (FUT) inside a climatic chamber, a photodetector followed by the proper signal processing instrumentation as well as the pulsed and the sinusoidal electric generators. With the climatic chamber HYGROS 15 the temperature and the humidity is carefully controlled on the FUT, which is placed under non-strain conditions to assure Brillouin independence from the strain effect.

The stimulated Brillouin scattering along the FUT is created by using two light waves traveling in opposite directions. The light from the laser diode is modulated in intensity by means of the electro-optic LiNbO3 Mach-Zehnder and amplified by the EDFA. The pulsed pump-wave is launched into the FUT by port 1 of the WDM to create the Stokes and anti-Stokes fields through the electrostriction effect. From the WDM (output port2) one side band (generated on the modulator by the sinusoidal RF signal) is launched into the end of the fiber element to stimulate the Brillouin scattering. When this side band matches the Stokes, then the scattered light is amplified.

*galindezca@unican.es; phone+34942 200877; fax +34942 200877 


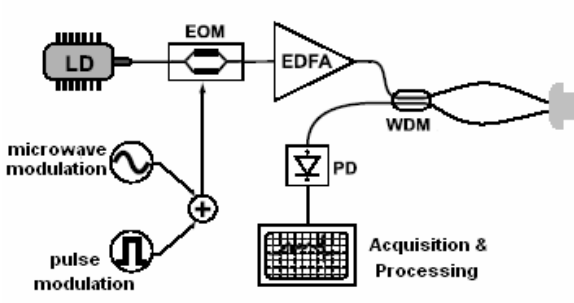

(a)

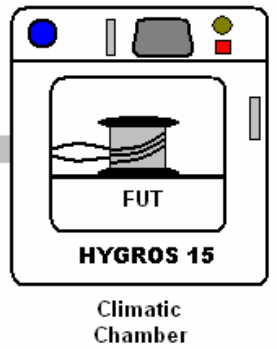

Chamber

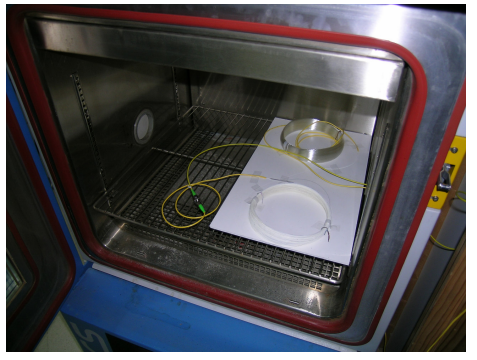

(b)

Fig. 1. Figure a) Experimental setup used to measure the shift on the Brillouin frequency. The laser source is in the $1.5 \mu \mathrm{m}$ wavelength range; EOM is the Electro optic Modulator Mach-Zehnder interferometer type; EDFA is the Erbium Doped Fiber Amplifier and the WDM selects the backscattered light. b) Picture of the optical fibers into the climatic chamber HYGROS 15 without strain or humidity due to reel.

By measuring the RF frequency corresponding to the amplified Brillouin peak, the Brillouin frequency shift is measured with high exactness and precision. The inspection of a desirable range on the fiber is carried by means of the travel time of the pump wave when it is sent as a train of pulses. With two meters of spatial resolution on four different pieces $(1000 \mathrm{~m}$ each) of monomode optical fibers measurements were realized. Frequency shift respect to humidity at constant temperature as well as its invariance at constant humidity. The behavior of the used fibers is listed in table 1 .

Table 1. Characteristics of optical fibers used in the experimental setup.

\begin{tabular}{|c|c|c|c|c|c|c|c|}
\hline Fiber & $\begin{array}{c}\text { Core diameter } \\
{[\mu \mathrm{m}]}\end{array}$ & $\begin{array}{c}\text { Clad + core } \\
\text { diameter }[\mu \mathrm{m}]\end{array}$ & $\mathbf{n}_{\text {core }}$ & $\Delta \mathbf{n}_{1}$ & $\Delta \mathbf{n}_{2}$ & $\begin{array}{l}\text { Fiber length } \\
\text { [m] }\end{array}$ & Index type \\
\hline Alcatel S3MC-AFC3 & 10 & 120 & 1.4655 & 0.005 & 0.0005 & 1000 & $\stackrel{\Delta \mathbf{n}_{1}}{u_{1}}$ \\
\hline Plasma Optical Fiber & 10 & 120 & 1.466 & 0.0042 & 0 & 1000 & $\Delta \mathrm{n}_{1}$ \\
\hline Standard monomode fiber & 10 & 120 & 1.460 & 0.002 & 0 & 1000 & $\Delta \mathrm{n}_{1}$ \\
\hline
\end{tabular}

\section{RESULTS AND ITS DISCUSSION}

The FUTs were first characterized in temperature. Results for the samples are shown in Fig 2. Also, in Fig. 3 it is possible to note the stability of the measurements along $2 \mathrm{Km}$ of optical fiber; these measurements were made for six different temperatures at $60 \%$ of relative humidity and with $2 \mathrm{~m}$ of spatial resolution.

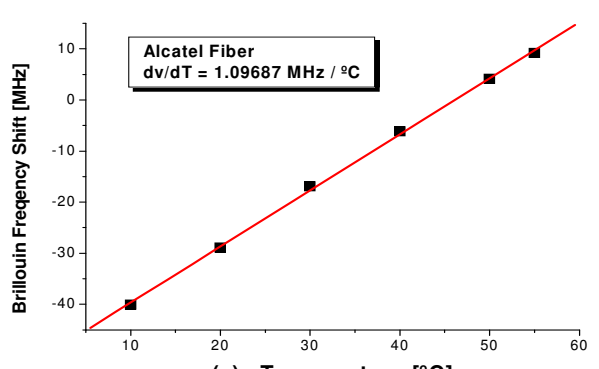

(a) Temperature [ํㅡ]

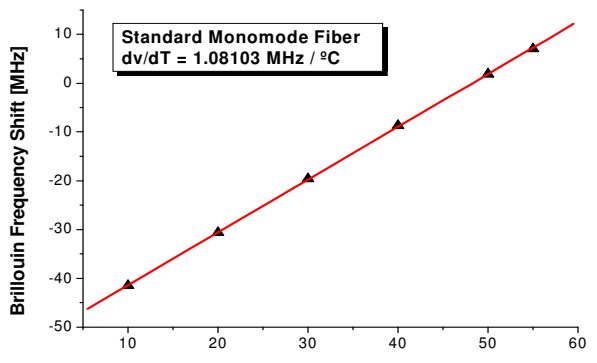

(b) Temperature $\left[{ }^{\circ} \mathrm{C}\right]$

Fig. 2. Experimental Brillouin frequency shift of the Brillouin gain spectrum as a function of temperature for (a) Alcatel fiber and (b) standard monomode fiber. The linear dependence between frequency shift and temperature is shown. 

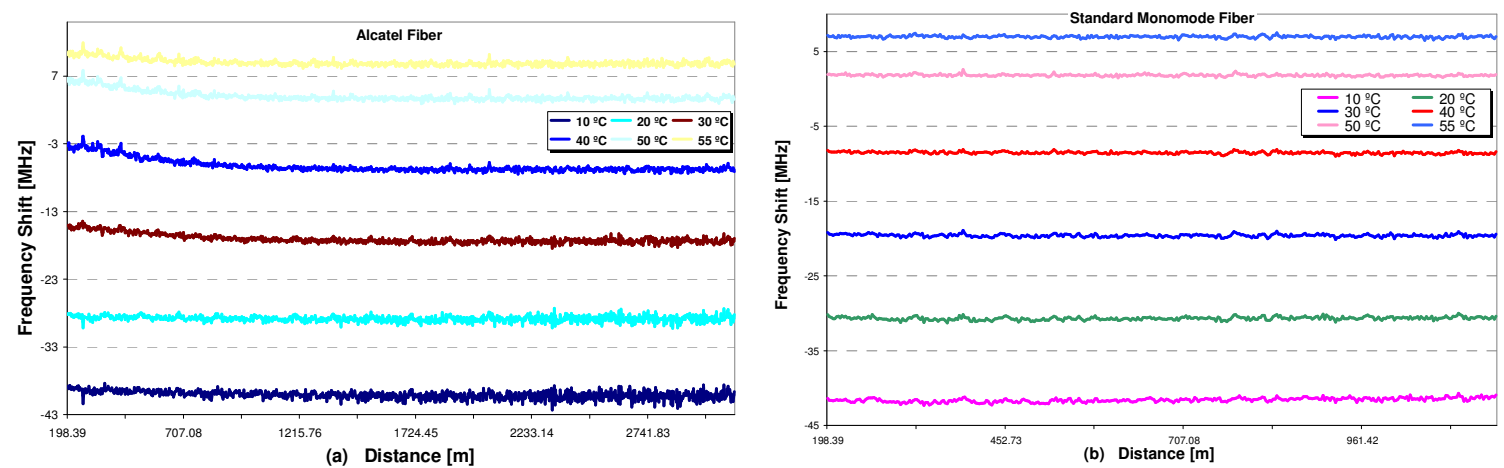

Fig. 3. Experimental Brillouin frequency shift along (a) $2 \mathrm{Km}$ of Alcatel fiber and (b) $1 \mathrm{Km}$ of standard monomode fiber.

When the fiber is placed into the climatic chamber at constant temperature and the relative humidity is varied between $40 \%$ and $80 \%$, a difference (step) in frequency is noticed. Fig. 4 shows the frequency shift stepped during three intervals of time; in each period of time the humidity is different $(40 \%(\bullet), 60 \%(\bullet)$ and $80 \%(\boldsymbol{\Delta}))$.

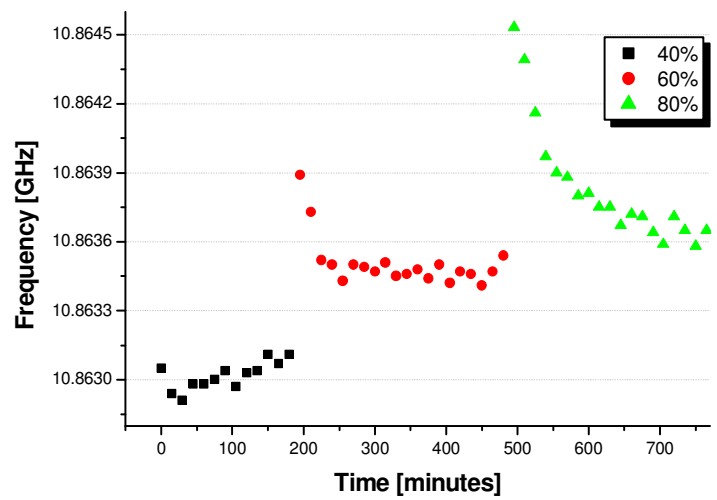

(a) Standar Monomone Fiber

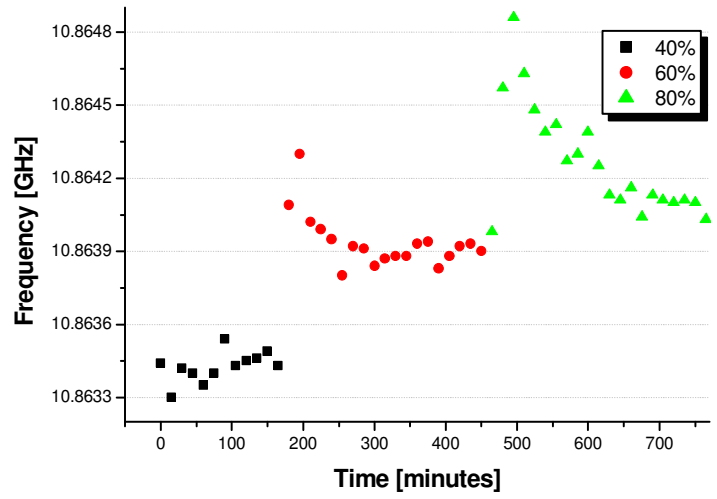

(c) Standar Monomone Fiber 2

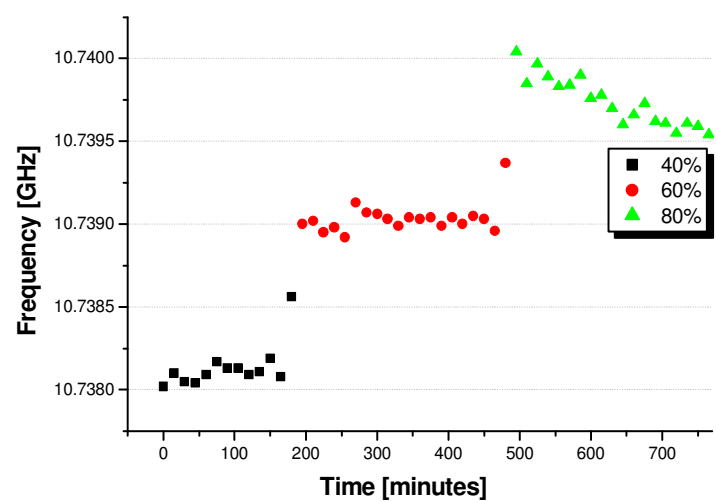

(b) Plasma Optical Fiber

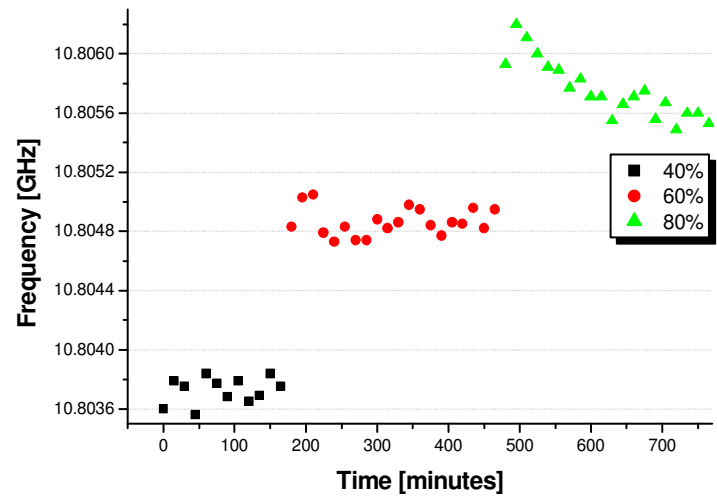

(d) Alcaltel S3MC Fiber

Fig. 4. Experimental Brillouin frequency shift of the Brillouin gain spectrum as a function of time for standard fiber (a) \& (c), Plasma optical fiber (b) and S3MC fiber at constant temperature (set at $25^{\circ} \mathrm{C}$ ) and $40 \%(\boldsymbol{\bullet}), 60 \%(\bullet)$ and $80 \%(\boldsymbol{\Delta})$ of humidity. The fiber is $1000 \mathrm{~m}$ length and the experiment has an approximately duration of 13 hours. 
The overshoots that appear at the beginning of any change on humidity correspond to variations of temperature related to the stabilization of the climatic chamber. However, the experiment was carried out considering the time that the chamber takes to stabilize. Then, the chamber can be steady most of the time that is assigned to a specific humidity. This fact can be appreciated in Fig. 4 where the points show a linear tendency at the stabilized relative humidity. The frequency shift is $0.5 \mathrm{MHz}$ larger at $60 \%$ than at $40 \%$ of humidity; whereas it is $0.2 \mathrm{MHz}$ from $60 \%$ to $80 \%$ of humidity. The difference between $40 \%$ and $60 \%$ in the frequency shift for the Plasma optical fiber is $0.9 \mathrm{MHz}$, from $60 \%$ to $80 \%$ is $0.6 \mathrm{MHz}$, finally in the S3MC fiber the deference of frequency shift is $1.1 \mathrm{MHz}$ for a humidity that varies from $40 \%$ to $60 \%$ and $0.8 \mathrm{MHz}$ for a variation of $60 \%$ to $80 \%$ in humidity.

\section{CONCLUSIONS}

In this paper the effects of humidity on Brillouin frequency shift in a controlled ambiance were reported. The temperature was set as a constant and it was only varied the humidity along the time. The relative humidity of the environment where the optical fiber is immersed, clearly affects the Brillouin frequency shift as it was illustrated in Fig. 4. It shows a non-linear variation in frequency shift for linear changes of humidity. The difference in the value of the Brillouin frequency shift depends as the optical fiber as the set humidity. When the humidity is increased to a high value $(80 \%)$ the difference on the frequency shift is less than the changes due to a step from a low humidity to a moderate one $(60 \%)$. Finally, it could conclude that the measured temperature using Brillouin scattering will show a larger uncertainty by effect of humidity.

\section{ACKNOWLEDGMENTS}

The authors acknowledge the support from the Spanish Government under the research projects TEC2004-05936-CO202 and TEC2007-67987-C02-01.

\section{REFERENCES}

J.M. Lopez-Higuera, E., Hand book of fibre optics sensing technology. 2002: Wiley \& Sons.

2 M. Niklès, L. Thévenaz, P.A. Robert. "Simple distributed fiber sensor based on Brillouin gain spectrum analysis". Opt. Lett. Vol 21, p 758-760. 1996.

3 Boyd, R.W., Non linear Optics. Second ed. 2003, USA: Academic Press; Elsevier Science.

4 K. Hotate, T. Hasegawa, "Measurement of Brillouin gain spectrum distribution along an optical fiber using a correlation based technique-proposal, experiment and simulation”, IEICE Trans. Electron E83 (3), 8, 2002. 